\title{
Responses to emerging and re-emerging infectious diseases: One world, One health
}

\author{
Jianqing $\mathrm{Xu}(\bowtie)^{1}$, Jianguo $\mathrm{Xu}(\bowtie)^{1,2}$ \\ ${ }^{1}$ Shanghai Public Health Clinical Center, Fudan University, Shanghai 201508, China; ${ }^{2}$ State Key Laboratory of Infectious Disease \\ Prevention and Control, National Institute for Communicable Disease Control and Prevention, Chinese Center for Disease Control and \\ Prevention, Beijing 102206, China \\ (C) Higher Education Press and Springer-Verlag GmbH Germany, part of Springer Nature 2018
}

During the past few decades, China has experienced rapid economic development, lifestyle changes, environmental deterioration, rapid urbanization, and population aging. After the epidemic of severe acute respiratory syndrome, Chinese government has invested heavily in disease control and prevention. However, the overall incidence of noticeable infectious diseases has increased rather than decreased. Emerging communicable diseases continue to threaten the country and the society. As a country with a huge population, high geographical and climatological diversity, the Chinese lesson and experience should have implication for global infectious diseases control and prevention.

The seasonal migration of birds has resulted in the spread of avian influenza viruses; the highly pathogenic H5N1 can directly cause diseases in local animals or humans and H7N9 can lead to the development of new recombinants with local viruses after setting down and then initiate to circulate in the local ecological environment. The natural connection of the world by migratory birds has established the geographic concept of "One world." The intensified economic activities and population migration have further strengthened "One world" and fueled the spread of pathogens (such as pandemic H1N1 2009, MERS, and Ebola). In this regard, it is critical to tackle with emerging and re-emerging infectious diseases by orchestrated global efforts, testified by this issue to bring scientists from China, Russia, USA and Australia together.

The second concept of "One world" is the coexistence and interaction between humans and microbes. Microbes and humans co-exist in one ecological environment, and microbes may enter the human body through food, breath,

Correspondence: Jianqing Xu, xujianqing@shphc.org.cn; Jianguo Xu, xujianguo@icdc.cn or direct contact among hosts (animal-to-human, water-tohuman, air-to-human, human-to-human, and mother-tobaby). An example of historic catastrophic events is the pneumonic plague epidemic that occurred between 1910 and 1911; in this epidemic, the pathogens were transmitted from marmots to humans due to large-scale hunting and trading of this specific animal with life-threatening Yersinia pestis. This outbreak was finally controlled by highly organized efforts headed by a famous doctor and national hero named $\mathrm{Wu}$ Lien-Teh (Zhang et al., this issue). This event reminds us the importance not only of ecology and human behavior but also monitoring pathogens in our ecological environment. Several works monitored pathogens in food (Liu et al., this issue; Wang et al., this issue); examples of such works are those conducted on Cyclospora cayetanensis (Jiang et al., this issue), on TB (Yang and Gao, this issue; Liu et al., this issue), on fungus in China (Chen et al., this issue) and in Russia (Vasilyeva et al., this issue), on viruses (Liu et al., this issue), and, importantly, on HBV in mothers (Shi et al., Front Med 2017, 11(4): 548-553). Microbes can also exist within humans; the interaction between microbes and host tissue cells/immune cells will drive their evolution, resulting in the clearance of pathogens, pathogen mutations, or pathogenesis (Yan et al., Front Med 2017, 11(4): 536-547). In addition, tools for precisely reading the outcome of interaction are critical, as reported in the report on the potential use of serum HBV RNA in antiviral therapy for chronic hepatitis B (Lu et al., Front Med 2017, 11(4): 502-508).

The "One world" concept indeed requires "One health" to match as an effective approach to caution against the danger of traveling to epidemic areas, thereby reducing the possible spread of infectious microbes, isolating infected subjects, controlling contaminated food (Liu et al., this issue; Wang et al., this issue), and implementing the most efficient approach, called the "vaccination of un-infected population." Thus, controlling infectious diseases by using 
vaccines or developing novel vaccines is vital. Current vaccination effort has focused on alleviating HBV (Zhang et al., Front Med 2017, 11(4): 490-501). New vaccine development strategies may be established based on natural infection of influenza (Stadlbauer et al., Front Med 2017, 11(4): 471-479; Nüssing et al., this issue) and HIV (Gao et al., Front Med 2017, 11(4): 480-489). In addition, blocking viral entry into host cells through human-derived antibodies (Jin et al., Front Med 2017, 11 (4): 462-470) or interrupting the life cycle by smallmolecule viral inhibitors (Wang et al., Front Med 2017, 11 (4): 449-461) has been intensively explored to contain emerging infectious diseases (such as SARS, MERS, Ebola, and influenza).

Finally, several new tools for surveillance of TB and bacterial diseases should be given attention. The first tool is a cost-effective regimen for TB testing; these regimens include traditional tuberculin skin testing (TST) and
QuantiFERON-TB Gold assay (QFT) (Li et al., Front Med 2017, 11(4): 528-535). TST can be used for TB screening, and QFT can be applied to confirm TB infection. QFT assay is expensive and specific, whereas TST is cost-effective but less specific. The second tool is genomic sequencing, which allows the precise diagnosis of a new infection from re-activated TB and the establishment of reliable transmission link in prevalence regions; this tool improves the assessment of outbreaks (Yang and Gao, this issue; Kan et al., this issue). Moreover, genomic sequencing will be greatly appreciated when developed to be cost effective.

\section{Compliance with ethics guidelines}

Jianqing $\mathrm{Xu}$ and Jianguo $\mathrm{Xu}$ declare no conflict of interests. This manuscript does not involve a research protocol requiring approval by the relevant institutional review board or ethics committee. 